\title{
A Novel Monochromator with Double Cylindrical Lenses
}

\author{
Takashi Ogawa, Boklae Cho, Sang Jung Ahn
}

Center for Advanced Instrumentation, Division of Industrial Metrology, Korea Research Institute of Standards and Science, Daejeon, Republic of Korea

Recently, various types of monochromators (MCs) have been proposed for advanced instruments such as the (scanning) transmission electron microscope ((S)TEM) [1,2], the scanning electron microscope (SEM) [3], and microscopes that utilize electron energy loss spectroscopy (EELS). There are several advantages with regard to the use of MCs. First, these devices offer improvements in the spatial resolution in low-energy regimes by reducing the contribution of chromatic aberrations. Second, they offer improvements in the energy resolution of the EELS spectra. Further improvements are necessary to reveal new information about specimens, such as the phonon signals. In addition, industry applications require simple and robust structures for MCs. In this study, we propose a novel MC with a high energy resolution and a simple structure. It consists of two electrostatic cylindrical lenses (CLs) in highly excited retarding mode, similar to a Möllenstedt energy analyzer [4]. They are placed in mid-plane symmetry and both are shifted from the optical axis of a microscope. A detailed discussion of the MC is given below.

For this MC, an offset CL is a key component. We give an outline of its physical behavior. A CL consists of three electrodes, similar to an Einzel lens but with rectangular openings in the center. This offers a stronger focusing effect in the $\mathrm{X}$ direction of the shorter side of the openings and a weaker focus in the $\mathrm{Y}$ direction. The electrons travel in the $\mathrm{Z}$ direction. The electrostatic potential of a CL can be expressed as follows:

$$
\Phi(x, y, z) \cong \phi(z)-\phi^{\prime \prime}(z) x^{2} / 4+\cdots .
$$

Ohiwa introduced the idea of a moving objective lens (MOL), which shows that superposition of a deflection field on a lens field is equivalent to displacement of the lens [5]. If the displacement of a CL is $X_{\mathrm{d}}$, the potential of the offset CL is given by

$$
\Phi\left(x-X_{d}, y, z\right) \cong \Phi(x, y, z)-X_{d} \frac{\partial \Phi}{\partial x}+\cdots \cong \phi(z)-\frac{\phi^{\prime \prime}(z) x^{2}}{4}+X_{d} \frac{\phi^{\prime \prime}(z) x}{2}+\cdots .
$$

The third term corresponds to the deflection effect caused by the displacement $X_{\mathrm{d}}$. The deflection function can be expressed as

$$
F_{1 x}(z)=X_{d} \phi^{\prime \prime}(z) / 2 .
$$

Smith and Munro derived a unified aberration theory for electrostatic lenses and multipoles [6]. We apply their theory to offset CLs. The paraxial ray equation can be written as follows:

$$
x^{\prime \prime}+\phi^{\prime} x^{\prime} / 2 \phi+\phi^{\prime \prime} x / 4 \phi=F_{1 x} / 2 \phi \text {. }
$$

Two independent, homogeneous solutions of Eq. 3 can be written as an axial ray $x_{\mathrm{a}}$ and a field ray $x_{\mathrm{b}}$. The inhomogeneous form of Eq. 3 can be solved by the variation of parameters method. The solution $x_{\mathrm{c}}$ can be written as

$$
x_{c}(z)=\frac{x_{a}(z)}{\sqrt{\phi\left(z_{0}\right)}} \int_{Z_{o}}^{z} \frac{F_{1 x}(\zeta) x_{b}(\zeta)}{2 \sqrt{\phi(\zeta)}} d \zeta-\frac{x_{b}(z)}{\sqrt{\phi\left(z_{0}\right)}} \int_{z_{o}}^{z} \frac{F_{1 x}(\zeta) x_{a}(\zeta)}{2 \sqrt{\phi(\zeta)}} d \zeta .
$$

Eq. 5 shows that defection function $F_{1 \mathrm{x}}$ produces deflection ray $x_{\mathrm{c}}$. The energy deviation, $\Delta \phi$, causes displacement $\delta x_{\mathrm{c}}$ to the original position of the trajectories. Here, $\delta x_{\mathrm{c}}$ includes not only the axial and transverse chromatic aberrations of the CL but also the displacement $x_{\mathrm{\kappa}}$ for deflection ray $x_{\mathrm{c}}$, which takes

$$
x_{\kappa}=C \Delta \phi x_{c}\left(z_{i}\right) / \phi\left(z_{i}\right) \text {, where } C=\left|\frac{\sqrt{\phi}}{x^{\prime} x_{c}}\right|_{z_{i}} \int_{z_{o}}^{z_{i}} \frac{x_{a} x_{c}{ }^{\prime \prime}}{\sqrt{\phi}} d z
$$


If we adopt this deflection ray $x_{\mathrm{c}}$ as an optical axis, the displacement $x_{\mathrm{\kappa}}$ can be considered as the energy dispersion to the optical axis by the energy deviation $\Delta \phi$. The common expression of energy dispersion is $D_{\kappa}=x_{\kappa} / \Delta \phi$. This discussion shows that the offset CL has an additional deflection effect and that this causes the energy dispersion. This enables the adoption of the offset CLs as deflection units for the MC. Next, we analyze the MC in detail by direct ray trace methods. We use EO-3D software by MEBS Ltd. to calculate the trajectories in three dimensions. The detailed dimensions of the CLs are as follows: the thickness of the electrodes is $10 \mathrm{~mm}$, the gap between the electrodes is $10 \mathrm{~mm}$, the shorter side of the opening is $10 \mathrm{~mm}$, and the longer side of the opening is $100 \mathrm{~mm}$. Figure 1 shows the schematics of the $\mathrm{MC}$ and the rays. The figure shows two cylindrical lenses $\left(\mathrm{CL}_{1}, \mathrm{CL}_{2}\right)$, two round transfer lenses $\left(\mathrm{TL}_{1}\right.$, $\mathrm{TL}_{2}$ ), two apertures, and an electron emitter. All the CLs and the TLs are arranged in mid-plane symmetry with $Z_{2}$. The two CLs are equally excited, with the same focal length of $f_{\mathrm{c}}$. The centers of CL and $\mathrm{CL}_{2}$ are separated by $2 f_{\mathrm{c}}$. Both of the CLs are offset by $X_{\mathrm{d}}$ from the optical axis of a microscope in the $\mathrm{X}$ direction, corresponding to the shorter side of the rectangular openings of the CLs. The two values $f_{\mathrm{c}}$ and $X_{\mathrm{d}}$ are related to the excitations of the CLs. Later discussions adopt $f_{\mathrm{c}}=40 \mathrm{~mm}$ and $X_{\mathrm{d}}=0.407$ $\mathrm{mm}$, where the center electrode voltages of the CLs $\left(V_{\mathrm{CL}}\right)$ are $-0.248 \mathrm{kV}$ from the emitter. The MC has two apertures. One is the entrance aperture, which is located in the front focal plane of $\mathrm{CL}_{1}\left(Z_{1}\right)$ to limit the incident angles of the emitted electrons. The other is the energy selection aperture, which is located at $Z_{2}$ to select the energy-dispersed rays. On the front side, the electron emitter is placed at $Z_{0}$. $T L_{1}$ and $\mathrm{TL}_{2}$ are arranged along the optical axis of the microscope to achieve stigmatic and dispersion-free imaging of the emitter at the exit plane $\left(Z_{4}\right)$ of the $\mathrm{MC}$. $\mathrm{TL}_{1}$, whose focal length is $f_{1}$, is located at $Z_{0}+f_{1}$ to collimate the incident rays from the emitter. $T L_{2}$ with a focal length of $f_{2}$ is placed at $Z_{3}+f_{2}$ to focus the rays on $Z_{4}$. The lens effects of the two TLs are identical in the X and Y directions.

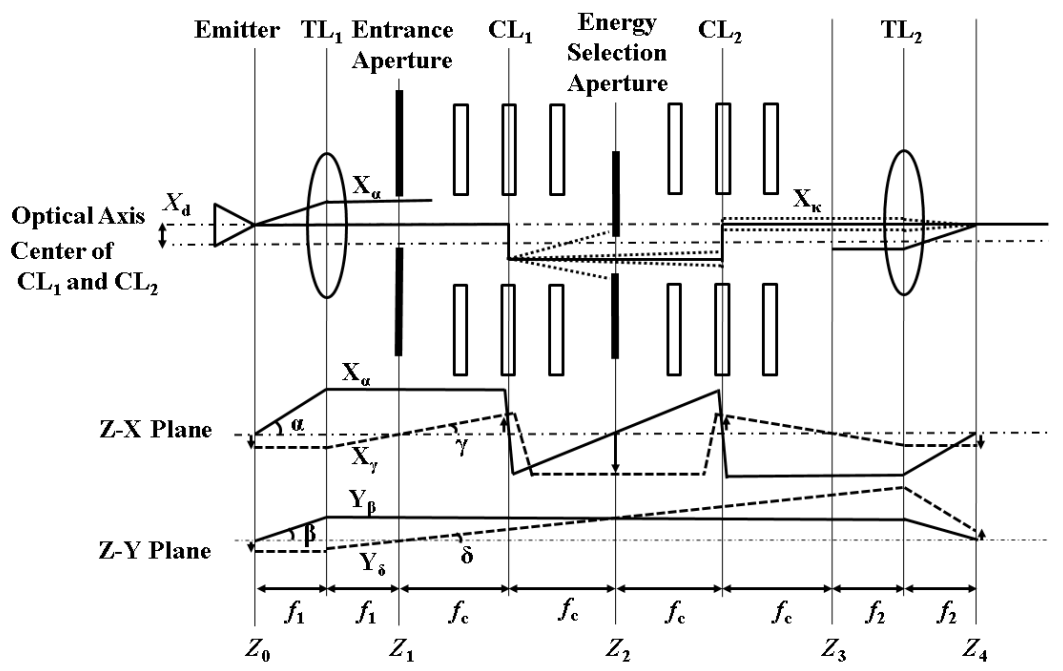

Figure 1. Schematics of the MC and the rays. The definitions of the planes $\left(Z_{0}-Z_{4}\right)$ and focal lengths $\left(f_{\mathrm{c}}\right.$, $\left.f_{1}, f_{2}\right)$ are given.

The total optics of the MC becomes symmetric with the middle plane $Z_{2}$. In the region of $Z_{0}-Z_{1}$, the rays are identical in the $\mathrm{X}$ and $\mathrm{Y}$ directions. However, the optical paths in the two directions differ in the region of $Z_{1}-Z_{3}$ because the CLs have astigmatic focusing effects. Figure 2 shows the results of ray trace simulations in this region. The primary energy $E_{0}$ of the electron beams is $4 \mathrm{keV}$. Figure $2 \mathrm{a}$ shows the optical axis in the $\mathrm{X}$ direction. The axis, which is offset by $X_{\mathrm{d}}$ to the center axis of the CLs, is deflected steeply at the center of $C_{1}$ and becomes parallel at $Z_{2}$. By succeeding $C L_{2}$, the axis is deflected back to the original axis at $Z_{3}$. The optical axis satisfies the condition of mid-plane symmetry with $Z_{2}$. 


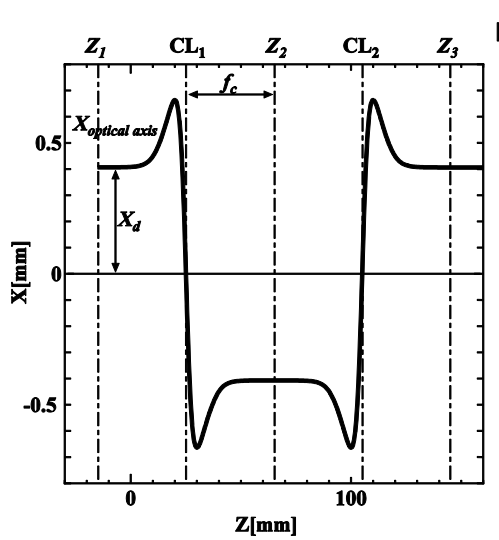

(a)

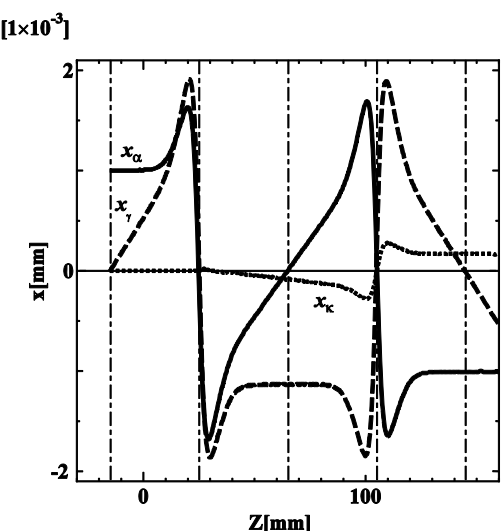

(b)

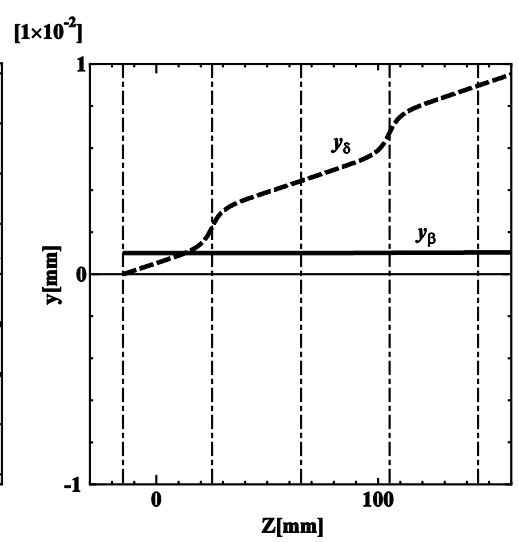

(c)

Figure 2. Simulated trajectories in the region of $Z_{1}-Z_{3}$, which is a part of the MC. (a) Optical axis. (b) $X$ fundamental rays and an energy dispersive ray. (c) Y fundamental rays.

Figure $2 \mathrm{~b}$ shows the fundamental rays $\left(X_{\alpha}, X_{\gamma}\right)$ and the energy dispersive ray $\left(X_{\kappa}\right)$ in the $X$ direction. The initial conditions are as follows: the offset distance of $X_{\alpha}$ is $1.0 \mu \mathrm{m}$, the inclined angle of $X_{\gamma}$ is $3.5 \mathrm{e}^{-5} \mathrm{rad}$, and the energy deviation of $X_{\kappa}$ is $1.0 \mathrm{e}^{-6}$. The $X_{\alpha}$ ray has three intermediate image planes and shows asymmetry with $Z_{2}$. The $X_{\gamma}$ ray has four image planes and shows symmetry with $Z_{2}$. In the $X$ direction, an object in $Z_{1}$ is transferred to an image in $Z_{3}$ at a magnification of -1 by the CLs. The dispersive ray $X_{\kappa}$ shows energy dispersion $D_{\kappa}$ of $23 \mu \mathrm{m} / \mathrm{eV}$ at $Z_{2}$. The $X_{\mathrm{\kappa}}$ ray becomes parallel to the optical axis and the $X_{\alpha}$ ray at $Z_{3}$, which means that the angular energy dispersion $(a \mid \kappa)$ vanishes there. The mathematic expression of $(a \mid \kappa)$ is based on the textbook [7]. Figure 2c shows the fundamental rays $\left(Y_{\beta}, Y_{\delta}\right)$ in the $\mathrm{Y}$ direction. The initial conditions are identical to those in the $X$ directions. Because the CLs have weak focusing power in the $\mathrm{Y}$ direction, the two rays have no intermediate image plane. The $Y_{\beta}$ ray remains straight and the $Y_{\delta}$ ray is slightly shifted twice to the off-axis direction at each center of the CLs without changing the angle. After passing the CLs, $\mathrm{TL}_{2}$ focuses the $X_{\alpha}$ and $Y_{\beta}$ rays stigmatically on the exiting plane $Z_{4}$, as shown in Figure 1. Also, $\mathrm{TL}_{2}$ focuses the $X_{\kappa}$ ray, which means that the lateral energy dispersion $(x \mid \kappa)$ vanishes at the exit of the MC. In total, the MC realizes optics with stigmatic and lateral dispersion-free imaging. Moreover, the emitter is imaged with a magnification of $|1|$ if the excitations of $\mathrm{TL}_{1}$ and $\mathrm{TL}_{2}$ are identical. The values of $f_{1}$ and $f_{2}$ are $10 \mathrm{~mm}$, as discussed below.

Next, we evaluate the performance of the MC. Figure 3 presents the aberration figures and Table 1 summarizes the characteristics of the MC. Figure 3 shows the beam distributions in the incident currents $I_{\text {in }}$ of 100,300 , and $1000 \mathrm{pA}$ at the middle plane $Z_{2}$ (upper figures) and at the exiting plane $Z_{4}$ (lower figures). In addition, different colors represent the energy conditions of $E_{0}, E_{0}+\delta_{\kappa} / 2$, and $E_{0}-\delta_{\kappa} / 2$, and each value of $\delta_{\mathrm{\kappa}}$ is summarized in Table 1. The other simulation conditions are as follows: Primary energy $E_{0}: 4 \mathrm{keV}$, excitation voltages of CLs $V_{\mathrm{CL}}:-0.248 \mathrm{kV}$, number of trajectories: 3000, emitter type: Schottky emitter, virtual source diameter: $28 \mathrm{~nm}$, angular current density: $500 \mu \mathrm{A} / \mathrm{sr}$, energy spread in FWHM: $0.833 \mathrm{eV}$ [8]. The aberration figures are constituted from a large number of trajectories whose initial conditions are randomly generated given the above simulation conditions. The energy selection slit widths $d_{\mathrm{s}}$ are calculated from the beam distributions at $Z_{2}$. The energy resolutions $\delta_{\mathrm{\kappa}}$ are calculated by the equation $\delta_{\mathrm{\kappa}}=2 d_{\mathrm{S}} / D_{\mathrm{\kappa}}$. The exiting currents $I_{\mathrm{out}}$ are calculated from the ratio of the electrons included in the energy range of $\delta_{\kappa}$ by postulating the energy distribution as a Gaussian with the energy spread described above. 

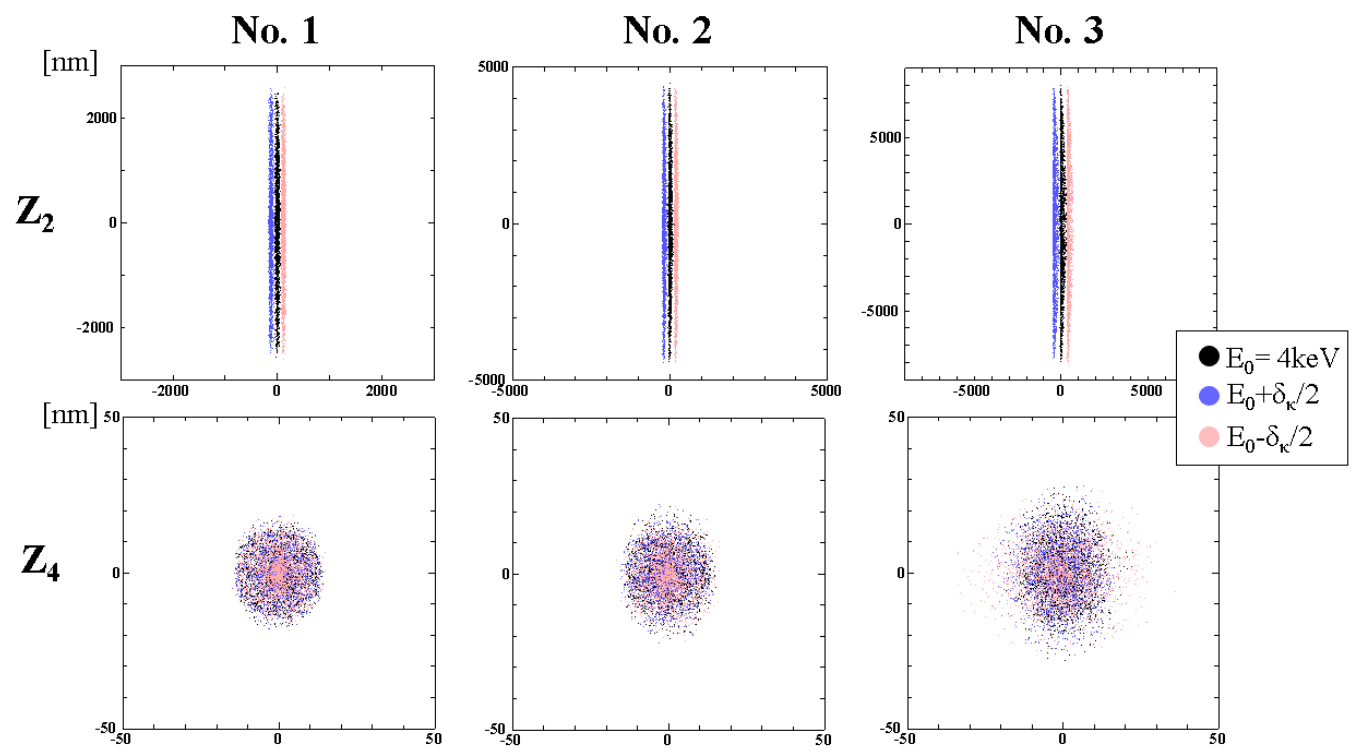

Figure 3. Aberration figures in the three incident currents conditions on the $Z_{2}$ and $Z_{4}$ planes.

\begin{tabular}{|c|c|c|c|c|}
\hline Condition & $\begin{array}{c}\text { Incident } \\
\text { current } \\
I_{\text {in }} \\
(\mathrm{pA}) \\
\end{array}$ & $\begin{array}{c}\text { Exit } \\
\text { current } \\
I_{\text {out }} \\
(\mathrm{pA}) \\
\end{array}$ & $\begin{array}{c}\text { Slit } \\
\text { width } \\
d_{\mathrm{s}} \\
(\mathrm{nm})\end{array}$ & $\begin{array}{c}\text { Energy } \\
\text { resolution } \\
\delta_{\kappa} \\
(\mathrm{meV}) \\
\end{array}$ \\
\hline No. 1 & 100 & 1.7 & 110 & 9.4 \\
\hline No. 2 & 300 & 7.1 & 180 & 15.4 \\
\hline No. 3 & 1000 & 50.4 & 390 & 33.5 \\
\hline
\end{tabular}

Table 1. The characteristics of the MC as calculated with Figure 3.

The upper figures in Figure 3 clearly show that the rays are line-focused and energy dispersive at the middle plane $Z_{2}$. By inserting slit-shaped apertures, an energy-selecting function is realized. Furthermore, the lower figures in Figure 3 show that the rays are focused stigmatically at the exit plane $Z_{4}$ due to their symmetry. Also, the different rays in terms of energy, as shown in different colors, are focused on the same position. This indicates that they are lateral dispersion-free, $(x \mid \kappa)=0$, at the exit plane $Z_{4}$. These results confirm that this optics can serve as a MC. In the smallest current condition of No. 1 , the beam diameter is about $30 \mathrm{~nm}$ at $Z_{4}$. Considering that the virtual source diameter is $28 \mathrm{~nm}$, this optics images the emitter with unit magnification at the exit. The energy resolution is better than 10 $\mathrm{meV}$. By increasing the beam current, the beam shapes begin to blur. The beam profiles at $Z_{2}$ become asymmetric in the $X$ direction and more triangular due to second-order aberrations. In contrast, the beam profiles at $Z_{4}$ show broad beam tails, but they remain symmetric in the $\mathrm{X}$ direction. These results imply that second-order aberrations are canceled and third-order aberrations are dominant on the exit plane $Z_{4}$. This is due to mid-plane symmetry of the optics. In the condition of No. 3, the energy resolution is approximately $34 \mathrm{meV}$. These results imply the potential of this optics as a high-performance MC.

We discuss the advantages and disadvantages of the MC with CLs by comparing it to other MCs. This $\mathrm{MC}$ is lateral dispersion-free, $(x \mid \kappa)=0$, at the exit plane $Z_{4}$, as shown in Figure 3. Therefore, this $\mathrm{MC}$ is better than single-stage MCs $[2,3,9]$. It can prevent rainbow illumination, which can cause limitations in 
the TEM operation conditions. However, the angular energy dispersion $(a \mid \kappa)$ of the MC remains at $Z_{4}$. This means that the energy dispersive ray $X_{\kappa}$ is inclined toward the optical axis at $Z_{4}$, as shown in Figure 1 . With regard to this point, the MC is inferior to an electrostatic $\Omega$-shaped MC [1] and a magnetic $\alpha$ shaped MC [10], where both $(x \mid \kappa)$ and $(a \mid \kappa)$ are canceled. This MC can be classified into the same category as MCs with multiple Wien filters [11-13], where $(x \mid \kappa)=0$ and $(a \mid \kappa) \neq 0$. From a different point of view, second-order aberrations of the $M C$ are canceled at $Z_{4}$ but not at the middle plane $Z_{2}$, as shown in Figure 3. The aberrations at $Z_{2}$ can deteriorate the energy resolution and cause non-isochromatic energy selection [14]. The $\Omega$-MC corrects the aberrations by curvatures of toroidal electrodes. The $\alpha-$ MC corrects them by hexapoles located in the front and back of the main prism. The multiple Wienfilter MCs cancel the aberrations at the exit, but not on the middle energy-selecting plane. With regard to the corrected ranks of aberrations, a MC with CLs can be classified into the same category as MCs with multiple Wien filters [11-13]. This is worse than sophisticated MCs [1,10] but better than single-stage MCs $[2,3,9]$. With the loss of its simplicity, this MC can be improved further by canceling aberrations with additional correctors. To cancel $(x \mid \kappa)$ and $(a \mid \kappa)$ at $Z_{4}$, the $X_{\kappa}$ ray should be parallel to the optical axis at the middle plane $Z_{2}$. This condition can be achieved by placing two quadrupoles at the regions of $\mathrm{CL}_{1^{-}}$ $Z_{2}$ and $Z_{2}-C_{2}$, similar to the $\alpha$-MC [10]. Canceling the second-order aberrations on the middle plane $Z_{2}$ can be achieved by placing two hexapoles at the regions of $Z_{1}-C_{1}$ and $C_{2}-Z_{1}$, similar to sector magnets $[7,10,14]$.

This MC has asymmetric internal optical paths, where the rays have three crossovers in the $\mathrm{X}$ direction but no crossover in the $\mathrm{Y}$ direction. This can avoid additional beam blur due to electron-electron interactions caused by stigmatic images with a high current density, similar to the $\Omega-\mathrm{MC}$ [1]. However, asymmetry of the beam profile at the middle plane $Z_{2}$ is greater than that of the $\Omega$-MC due to its weak focusing power in the $\mathrm{Y}$ direction. This may impose some difficulties in the manufacturing of the energy selection aperture due to the high aspect ratio. Imperfections in the aperture may cause an inhomogeneous current distribution in the $\mathrm{X}$ and $\mathrm{Y}$ directions of the exiting beams. The aperture can be fabricated using the latest nano-processing techniques, such as EB lithography or a focused ion beam. From a different standpoint, this MC utilizes CLs in retarding mode. At the centers of the CLs, lowenergy electrons are expected to be weak against disturbances such as external magnetic fields. This is also a disadvantage of the MC. For a practical design, double magnetic shields with high permeability, made of permalloy, should be implemented outside of the CLs. These are often adopted in low-energy electron analyzers.

It should be noted that a significant advantage of the $\mathrm{MC}$ is its simple optical configuration without multipole optics. Through the use of CLs as deflection elements, the mechanical structures, electronics, and control units can be simplified compared to other MCs with complicated optics. The $\Omega$-MC adopts four deflection units with eight toroidal electrodes, which have different curvatures in not only the horizontal but also the vertical sections. The curvatures are determined by a simulation to achieve focusing power in the $\mathrm{Y}$ direction and the compensation of second-order aberrations. To manufacture the electrodes, sophisticated machining techniques for 3D curved surfaces are necessary. Also, a high level of alignments of the electrodes in 3D is required. MCs with Wien filters adopt multipole units in several stages, which also require fine machining and the alignment of separate poles to achieve the expected performance level. The $\alpha$-MC adopts three main deflectors, 14 quadrupoles, and eight hexapoles. To control these units, a large number of power sources, which should be stable and low levels of noise, are necessary. Compared to these MCs, the MC with the CLs has a simple configuration. For CLs, rectangle openings in flat plate electrodes are necessary. Although they require accurate 
machining, this can be realized by conventional machining tools. The MC has an advantage in its assembly process because CLs consist of flat plate electrodes without separations in the azimuth directions. In addition, the MC requires only two electronic units for two CLs. The number of units is low compared to other MCs. This brings a benefit when the MC is used for the HV regions of electron guns. Two additional units are necessary for TLs, but they can be used as the condenser lenses of microscopes. These reductions of complexity are a great advantage for MCs. They lead to a highly stable, robust, and cost-effective means of realizing MCs, which are required by industry.

There are several other features of the proposed MC. It has pure electrostatic optics, which leads to good vacuum conditions suitable for electron guns. It provides a quick response and good reproducibility. Also, this MC has linear optics, which results in easy alignment compared to curved optics. It also offers the high mechanical stiffness of a microscopy column. Furthermore, this MC enables use of the central portion of the beams from the electron source. This differs from a MC with a modified condenser lens, which utilizes the off-axis region of the electron emitter [3]. This improves the stability of the currents and the symmetry of the beam profiles.

In conclusion, we propose a novel monochromator with double cylindrical lenses. They are operated in retarding modes, located in a symmetric condition on the middle plane, and offset to the axis of a microscope. The proposed MC includes two additional round transfer lenses and two apertures. On the mid-plane, energy filtering is realized. The calculated energy dispersion is $23 \mu \mathrm{m} / \mathrm{eV}$ and the estimated energy resolution is better than $10 \mathrm{meV}$ for $4 \mathrm{keV}$. On the exit plane, stigmatic and lateral dispersionfree imaging of the emitter with unit magnification is achieved. A comparison with other MCs clarifies the pros and cons of the MC. This MC has advantages in its simple structure and high performance. It can bring improvements in the spatial resolutions and energy resolutions of microscopes. In the future, we plan to build the MC and evaluate it in experiments. Further, we will pursue additional improvements by canceling higher aberrations with multipole correctors [15].

\section{References:}

[1] H Rose, Ultramicroscopy 78 (1999), p. 13.

[2] HM Mook and P Kruit, Ultramicroscopy 81 (2000), p. 129.

[3] A Henstra et al, Microsc. Microanal. 15 (S2) (2009), p. 168.

[4] G Möllenstedt, Optik 5 (1949), p. 499.

[5] H Ohiwa, J. Vac. Sci. Tech. 15 (1978), p. 849.

[6] MR Smith and E Munro, Optik 74 (1986), p. 7.

[7] H Wollnik in "Optics of Charged Particles", (Academic Press, London).

[8] GA Schwind et al, J. Vac. Sci. Tech. B 24 (2006), p. 2897.

[9] PC Tiemeijer, Ultramicroscopy 78 (1999), p. 53.

[10] OL Krivanek et al, Phil. Trans. R. Soc. A 367 (2009) p. 3683.

[11] M Mukai et al, Microsc. Microanal. 12 (S2) (2006) p. 1206CD.

[12] K Tsuno, Nucl. Instr. Meth. A 645 (2011) p. 12.

[13] E Plies et al, Nucl. Instr. Meth. A 645 (2011) p. 7.

[14] H Rose and D Krahl in "Energy-Filtering Transmission Electron Microscopy", ed. L Reimer, (Springer-Verlag, Heidelberg), p. 43.

[15] We are very grateful to Prof. T. Ichinokawa from Waseda University for suggesting the energy analyzers with two cylindrical lenses. Grants: 2011-0030233 and 2014R1A6B1A01048695. 